\title{
Going into reverse
}

\section{Reversible computation: how feasible is a computer that is both logically and physically reversible?}

\section{Seth Lloyd}

$\mathrm{N}$ othing in life is certain except death, taxes and the second law of thermodynamics. All three are processes in which useful or accessible forms of some quantity, such as energy or money, are transformed into useless, inaccessible forms of the same quantity. That is not to say that these three processes don't have fringe benefits: taxes pay for roads and schools; the second law of thermodynamics drives cars, computers and metabolisms; and death, at the very least, opens up tenured faculty positions.

Indeed, most of the good things in lifeincluding life itself — arise from this gradual degradation of the useful into the useless, of order into disorder, known in physical terms as an increase in entropy. Still, wouldn't it be nice sometimes to slow down or even momentarily stop this inevitable dissipation of resources? After all, the second law of thermodynamics doesn't require that entropy always increase: it leaves open the possibility that entropy can remain constant. There are some processes that are effectively reversible: no energy is dissipated, and entropy remains constant, or almost so. Chemical reactions are reversible if run sufficiently slowly; they can dissipate arbitrarily small amounts of energy per step. Coherent quantum-mechanical processes such as tunnelling and superconductivity are reversible and can operate without dissipation. And so can computation, in principle.

More than 40 years ago, Rolf Landauer realized that there was a fundamental connection between computation and the second law of thermodynamics. Just like physical processes, logical processes can be either reversible or irreversible. A logic operation is reversible if its inputs can be inferred from its outputs. For example, the NOT operation that takes 0 to 1 and vice versa is reversible because if $Y=$ NOT $X$, then knowing that $Y$ is 0 allows one to deduce that $X$ is 1 . By contrast, the ERASE operation, which always gives the output 0 regardless of its input, is irreversible: the output gives no knowledge of the input.

Landauer pointed out a strong connection between logical irreversibility and physical irreversibility. First, logically reversible operations such as NOT can be implemented using physically reversible operations. For example, if a proton spinning clockwise around some axis is taken to register YES,

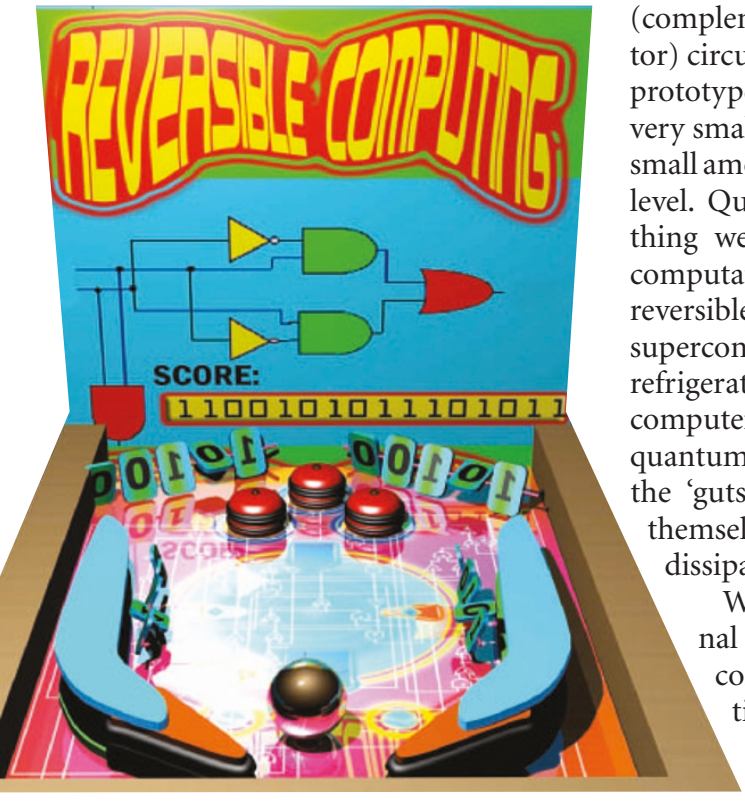

(complementary metal oxide semiconductor) circuits. Perhaps even more impressive, computers, even though amounts of entropy at the microscopic Quantum computers are the closest have to devices that carry out computation in a logically and physically reversible fashion. (Of course, the lasers, onducting magnets and dilution frigerators that are needed to run quantum computers generate plenty of heat. But the quantum logic operations that take place in the 'guts' of the quantum computer are themselves fundamentally reversible and dissipate almost no energy.)

What's the catch? In Bennett's original design and in reversible CMOS computers, the catch is that dissipation only goes to zero in the limit of zero speed. In the case of quantum computers, the catch, to paraphrase John Donne, is that no and one spinning anticlockwise registers $\mathrm{NO}$, then application of a magnetic field causes the proton to flip from YES to NO and back again, all without dissipation or increase in entropy. The flipping spin implements a NOT operation in a physically reversible way. Second, a logically irreversible operation such as ERASE requires physical irreversibility. A bit in your computer's memory is registered by the charge in a tiny capacitor: when you erase the bit by restoring the capacitor to an uncharged state, then half the time the energy in the capacitor is dissipated as heat.

Conventional computations are built up of both reversible and irreversible logical operations, so at first the idea that logical irreversibility requires physical irreversibility (which is known as Landauer's principle) seemed to imply that computation requires dissipation. But then Charles Bennett and, independently, Ed Fredkin realized that most logically irreversible operations can be embedded in slightly more complicated reversible operations. As a consequence, computation can in principle be implemented using reversible physical processes such as slow chemical reactions or coherent quantum processes. The one logically irreversible process that cannot be embedded in a more complicated reversible process is erasure: when you erase the last copy of a bit from your computer, entropy must increase elsewhere.

In the past decade, logically and physically reversible computations have been implemented using logically reversible CMOS qubit is an island, entire unto itself. No matter how weak a qubit's interactions with the rest of the Universe may be, sooner or introduce an error into the computation.

Like added entropy, an error consists of unwanted information. Computers can be fitted out with error-correcting codes that seek out errors and correct them, by erasing the erroneous information. By Landauer's principle, erasure of a bit in the computer requires entropy increase elsewhere. For computers, error is the ultimate source of entropy.

Of course, slow computations full of errors can be performed with as little dissipation as you like. But the second law says that if you want to compute fast and accurately, you have to dissipate.

What about death? Must computation, like all physical processes, grind to a close? In fact, as long as the expansion of the Universe keeps on supplying free energy and environment into which to reject errors, the known laws of physics apparently allow a computer to compute for ever. Just what might an eternal computer compute? The answer will have to wait.

Seth Lloyd is in the Department of Mechanical Engineering, Massachusetts Institute of Technology 3-160, Cambridge, Massachusetts 02139, USA.

\section{FURTHER READING}

See articles by R. Landauer, C. H. Bennett and S. Lloyd in Leff, H. S. \& Rex, A. F. (eds) Maxwell's Demon 2: Entropy, Classical and Quantum Information, Computing, 2nd edition (Institute of Physics, Bristol, 2003). later one of them will flip that qubit and 